\title{
Comprehensive Forecasting Method of Monthly Electricity Consumption Based on Time Series Decomposition and Regression Analysis
}

\author{
Luan, Changfeng; Pang, Xinfu; Wang, Yanbo; Liu, Li; You, Shi
}

Published in:

Proceedings of $2<$ sup $>$ nd $</$ sup $>$ International Conference on Industrial Artificial Intelligence

Link to article, DOI:

10.1109/IAI50351.2020.9262169

Publication date:

2020

Document Version

Peer reviewed version

Link back to DTU Orbit

Citation (APA):

Luan, C., Pang, X., Wang, Y., Liu, L., \& You, S. (2020). Comprehensive Forecasting Method of Monthly Electricity Consumption Based on Time Series Decomposition and Regression Analysis. In Proceedings of $2^{\text {nd }}$ International Conference on Industrial Artificial Intelligence IEEE. https://doi.org/10.1109/IAl50351.2020.9262169

\section{General rights}

Copyright and moral rights for the publications made accessible in the public portal are retained by the authors and/or other copyright owners and it is a condition of accessing publications that users recognise and abide by the legal requirements associated with these rights.

- Users may download and print one copy of any publication from the public portal for the purpose of private study or research.

- You may not further distribute the material or use it for any profit-making activity or commercial gain

- You may freely distribute the URL identifying the publication in the public portal 


\title{
Comprehensive Forecasting Method of Monthly Electricity Consumption Based on Time Series Decomposition and Regression Analysis*
}

\author{
Changfeng Luan, Xinfu Pang, Member, IEEE, Yanbo Wang, Li Liu, Member, IEEE, Shi You, Member, IEEE
}

\begin{abstract}
Power consumption prediction is the basis of implementing planned power consumption and preparing production plan. It is one of the main projects in the design of industrial and mining enterprises. It is also an important link to ensure the balance between national economic needs and power supply. Due to the influence of distributed energy and the change of power demand and load characteristics of the user side compared with the past, the power consumption prediction starts to face smallscale users and is more easily disturbed by various influencing factors, so the traditional prediction method is not fully suitable for today's power consumption prediction. Firstly, STL is used to decompose the power consumption sequence of corresponding month into trend component, season component and random component. Secondly, the BP neural network model is used to predict the seasonal component of the month when the seasonal mutation and major festivals are located. ARIMA model is used to predict the trend component. The average value is used to predict the random components. Then, the predicted values of the three components are reconstructed into the final predicted values. Finally, the algorithm is compiled by $R$ language, and the validity of the proposed method is verified by the actual monthly electricity sales data of a University Park in the north. And further consider the prediction method of economic factors.
\end{abstract}

Key Words: monthly electricity consumption forecasting; electricity consumption characteristics; personalized decomposition; time series; STL model

\section{INTRODUCTION}

\section{A. Literature Review}

Monthly electricity consumption not only changes with time, but also relates to economic, political, human activities and other influencing factors. Therefore, when considering the influence of influencing factors on electricity consumption comprehensively, not only the change rule of user's electricity consumption characteristics with time, but also the influence of external influencing factors on electricity consumption should be considered.

Due to the open competition of electricity market restructuring, the monthly electricity consumption forecasting

*Reseach supported by the National Natural Science Foundation of China (61773269), Overseas Training Project of Liaoning Colleges and Universities (2018LNGXGJWPY-YB034), the Program for Liaoning Excellent Talents in University (LR2019045), the Natural Science Foundation of Liaoning Province of China (2019-KF-03-08, 20180550508), the Program for Shenyang High Level Innovative Talents (RC190042).

C.F. Luan, X.F. Pang and L. Liu are with Key Laboratory of Energy Saving and Controlling in Power System of Liaoning Province, Shenyang Institute of Engineering, Shenyang, China (Corresponding author : E-mail address: pangxinfu@163.com(X.F. Pang)).

Y.B. Wang is with State Grid Dandong Electric Power Supply Company, Dandong, China.

S. You is with Department of Electrical Engineering, Technical University of Denmark, Lyngby, Denmark. begins to face small-scale users, and the impact of randomness on the forecasting results is greatly increased, so the traditional load forecasting cannot be fully applied to the electricity consumption forecasting.

Extensive research on prediction methods has been carried out in the references. Literature [1-3] proposed a method of electricity forecasting considering the time lag of economic factors on load, which reflects the changes of economic situation on electricity forecasting. Literature [4] proposed a decomposition model using moving regression and smooth spline as smoothing method is proposed to decompose the time series of power demand, and two neural networks are trained to predict the decomposition results respectively. The results show that the sequence decomposition prediction is more accurate than the sequence direct prediction. Literature $[5,6]$, the time series is decomposed into trend component and season component by classical decomposition model, and each component is predicted by SARIMA (seasonal autoregressive integral moving average) and weighted method respectively. Literature $[7,8]$, the X12 multi-plication model is used to decompose the electric quantity series and establish different models to predict each component. The X12 model overcomes the disadvantage that the trend values of several samples at the beginning and end of the classical decomposition model are difficult to be estimated. Literature [9], ARIMA (autoregressive integral moving average) model, x12-arima model and polynomial regression are used to predict the monthly error number of software, which verify the superiority of x12arima model, but X12 model still has the limitations that it can only process quarterly or monthly data and cannot control the change rate of seasonal components. Literature [10], the influence of economic disturbance on power consumption is considered, and x12-arima model is used to predict the final power consumption by coupling economic factors and power consumption series. The prediction results of this method are more accurate, but the data requirements of economic factors are strict.

\section{B. Motivation}

In the prediction of monthly electricity consumption, the previous research method is to directly model and predict the time series of historical data. For example, according to the time series characteristics of electricity consumption, without considering the influence of many factors, ARIMA model is established according to the past change law of electricity consumption, and the conventional linear regression prediction is carried out for monthly electricity consumption, or only considering the influence of seasonal factors on monthly electricity consumption, through the seasonal difference The SARIMA model is established to predict the monthly electricity consumption. All 
these methods ignore that the time series of monthly electricity consumption often contains components with different characteristics in the actual forecasting process.

Based on this, this paper analyzes the internal variation of power consumption time series with time, and proposes a comprehensive monthly power consumption prediction method based on STL model. Firstly, the monthly electricity consumption is decomposed into trend component, seasonal component and random variable by STL. ARIMA model is used to predict trend component. For seasonal component of non-seasonal sudden change month, historical same period value is taken as prediction value directly. For seasonal sudden change month, BP neural network is used to predict. The random component is expressed by historical average value. Then, the prediction value is reconstructed. Finally, further considering the influence of economic factors, the trend component of economic data is extracted by $\mathrm{X} 12$ decomposition model, and the two trend components are fitted by VAR model, and the prediction methods of seasonal component and random component are unchanged.

\section{PROBLEM DESCRIPTION}

With the access of distributed energy, the power demand and load characteristics of the user side will change accordingly; the reorganization of the open competition power market makes the monthly power consumption forecast start to face the small-scale users, and the randomness will greatly increase the impact on the forecast results; the performance appraisal system of the electricity sales market puts forward higher requirements for the accuracy of the monthly power consumption forecast.

\section{A. Classical Prediction Method}

An ordered sequence that records variable values over a fixed time interval creates a time series. Time series prediction is to predict the future value of a variable based on the previously observed value. Time series models are usually classified as topdown models and represent the relationship between variables and time. A typical example of a time series is the power consumption recorded at a fixed time interval to predict the power consumption in the next period.

Regression analysis determines the prediction function by calculating the dependent variable value of one or more independent variables. Regression analysis method can be divided into nonlinear regression model and linear regression model according to the structure of function about unknown coefficient.

Many of the above electricity forecasts have their own applicable conditions and limitations, power consumption forecasting itself is a complex non-linear problem, which is not only affected by season, user behavior and economic development, but also has the internal change rule of historical data.

\section{B. The Improvement of Prediction Method}

Using cluster analysis to analyze the load big data, combined with cluster technology to analyze the typical user's power consumption characteristics and its influencing factors, and then from the perspective of autocorrelation analysis and crosscorrelation analysis, provide ideas for monthly power consumption prediction. In view of the limitations of traditional time series model for direct prediction of monthly power consumption, this paper proposes a comprehensive prediction method based on time series decomposition and regression analysis, considering the different characteristics of power consumption series in different months. On the basis of considering the influence of season mutation and major holidays on monthly electricity consumption, the paper further considers the improvement measures of economic factors on monthly electricity consumption from the perspective of cross-correlation analysis, and puts forward the method of monthly electricity consumption prediction based on X12 and STL decomposition model.

\section{MODELS OF RELATED THEORIES}

\section{A. ARIMA Model}

Firstly, the nonstationary part of the time series is smoothed by the difference operation, and then the dependent variable, the lag value of the random error term and the present value of the random error term are regressed. In $\operatorname{ARIMA}(\mathrm{p}, \mathrm{d}, \mathrm{q})$ model, there are three parameters, $p, d$ and $q$, where $p$ represents the number of observations and q represents the number of residuals. In order to make the whole sequence become a stable sequence, the difference number $d$ is proposed. Each observation in the sequence is represented by a linear system of historical observations and residuals.

$$
\left\{\begin{array}{c}
Y_{t-k}=(1-B)^{d} \omega_{t} \\
Y_{t}=\mu+\beta_{1} Y_{t-1}+\beta_{2} Y_{t-2}+\ldots+\beta_{p} Y_{t-p}-\theta_{1} \varepsilon_{t-1}-\theta_{2} \varepsilon_{t-2} \ldots-\theta_{q} \varepsilon_{t-q}+\varepsilon_{t}
\end{array}\right.
$$

where, $\omega_{t}$ is the trend component of monthly electricity consumption; $B$ is the lag operator, $B_{k} \times \omega_{t}=Y_{t-k}$; the selection of difference times $\mathrm{d}$ is related to the actual sequence, which should not be too large, generally no more than $2 ; Y_{t}$ is the trend component sequence of stabilization; $\varepsilon_{t}$ is the residual of prediction; $\mu$ is the mean value of the sequence; $\theta_{1}, \theta_{2}, \ldots, \theta_{q}$ is the moving average coefficient; $\beta_{1}, \beta_{2}, \ldots, \beta_{p}$ is the autoregressive coefficient.

\section{B. SARIMA Model}

When the series presents the seasonal variation law with the period of quarter / month, the time series can be further transformed into the $(p, d, q) \times(P, D, Q)$ order SARIMA model with the change period as the order after the $D$-th seasonal difference.

$$
\Phi_{p}(L) A_{p}\left(L^{s}\right)\left(\Delta^{d} \Delta_{s}^{D} y_{t}\right)=\theta_{q}(L) B_{Q}\left(L^{s}\right) v_{t}
$$

where $\Phi_{p}(L)$ is a non seasonal autoregressive operator, $\Phi_{p}(L)=1-\Phi_{I}(L)-\Phi_{2}\left(L^{2}\right)-\ldots-\Phi_{p}\left(L^{p}\right) ; A_{p}\left(L^{s}\right)$ is a seasonal autoregressive operator, $A_{p}\left(L^{s}\right)=1-\alpha_{1} L^{s}-\alpha_{2} L^{2 s}-\ldots$ $\alpha_{p} L^{p s} ; \theta_{q}(L)$ is a non seasonal moving average operator, and $\theta_{q}(L)=1+\theta_{1} L+\theta_{2} L^{2}+\ldots+\theta_{q} L^{q} ; B_{Q}\left(L^{s}\right)$ is a seasonal moving average operator, and $L$ is a lagging operator; $B_{Q}\left(L^{s}\right)=1+\beta_{1} L+\beta_{2} L^{2 s}+\ldots+\beta_{Q} L^{Q s} ; D$ is the number of seasonal differences; $P$ is the lag order of seasonal autoregression and $Q$ is the lag order of seasonal moving average operator; $\Phi_{p}, \theta_{q}$ are the coefficients of $p$-order autoregression 
model and $q$-order moving average model respectively; $v_{t}$ is the white noise process.

\section{X12 Model}

The multiplication model assumes that the influence of each component on the development of the phenomenon is interrelated, based on the absolute quantity of the trend component, and the other components are expressed in the form of proportion. The monthly electricity consumption series is represented by the product of three components representing its trend factor, seasonal factor and random factor.

$$
Y=Y_{\text {trend }} \times Y_{\text {seasonal }} \times Y_{\text {random }}=Y_{t} \times Y_{s} \times Y_{r}
$$

where, $t$ is the number of samples; $X$ is the response variable; $Y$ is the prediction variable; $U$ is the regression error.

\section{Neural Network Method}

At present, one of the most widely used neural network models is BP network, which is a multilayer feedforward network trained by error back propagation algorithm. The net input value of the $j$ th neuron can be expressed as

$$
S_{j}=\sum_{i=1}^{N} w_{j i} \cdot x_{i}+b_{j}=W_{j} X+b_{j}
$$

where $b_{j}$ is the threshold value, $x_{i}$ is $1,2 \ldots . . \mathrm{n}$, $\mathrm{X}=\left[x_{1} x_{2} \ldots x_{n}\right]^{\mathrm{T}} ; W_{j}$ represents the link strength between the $i$ and $j$ neurons $W_{j}=\left[w_{j 1} w_{j 2} \ldots w_{j n}\right]$. If $x_{0}=1, " w_{j 0}=b_{j}$, then $\mathrm{X}=\left[x_{0}, x_{1}, x_{2}, \ldots x_{n}\right]^{\mathrm{T}}, W_{j}=\left[w_{j 0}, w_{j 1}, w_{j 2}, \ldots w_{j n}\right]$. So the net input of node $j$ can be expressed as

$$
S_{j}=\sum_{i=1}^{N} w_{j i} \cdot x_{i}=W_{j} X .
$$

\section{E. Algorithm Process}

(1) STL decomposition model flowchart

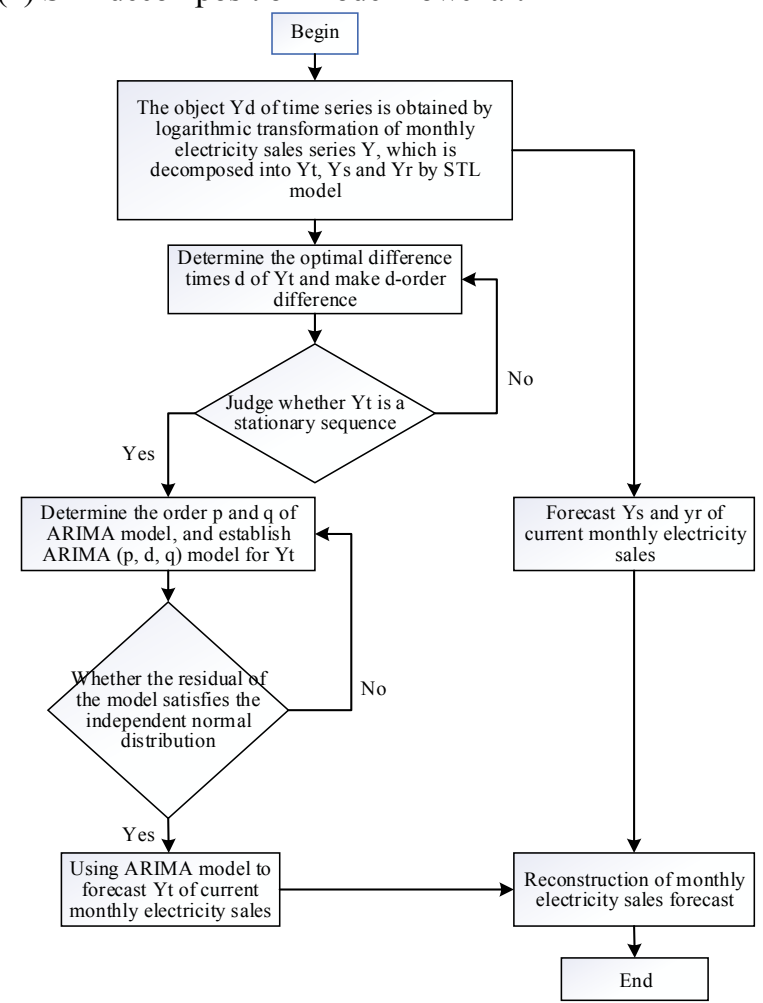

Fig.1. Monthly electricity consumption forecast model based on STL model

\section{(2) Basic steps of DIANA model}

Step1: Divide the given load data into a group, and find the cluster with the largest diameter from this group.

Step2: Select the average dissimilarity between this group and other load data points, that is, the largest load data point, and divide this point into the newly divided cluster, and put the rest into the original group.

Step3: Operate circularly. In the original cluster, find the closest point to the midpoint of the newly split group, which is not greater than the closest point of the midpoint of the original cluster, and add the point to the newly split group.

Step4: Until no new points of the original cluster are assigned to the newly split group, and the newly split cluster and the original group are the selected cluster, which is split into two groups, and then combined with other groups to form a new group set, until all the clusters are processed, the program is terminated.

(3) Basic steps of DBSCAN model

Step1: Select an unprocessed sample from the database.

Step2: If the data points extracted from the given data set are the key objects, then all the data points that can be reached from the point will be found, and then they will form a group.

Step3: If the extracted data point is not the key object, then it will not continue to work, and we will continue to process the points that have not been accessed.

Step4: If all the given data points are processed, the program will be ended.

\section{OPERATION RESULTS AND ANALYSIS}

\section{A. Classification of Loads}

Vertical BIC is Bayesian information. DIANA model needs to specify the optimal number of clusters in advance. The "mclust" package in $\mathrm{R}$ language can be used to complete the prediction of data information clustering data and the determination of the optimal amount, so that hierarchical clustering can be used for data mining and load imprinting extraction of a variety of data information. The pre-processing function can determine the optimal number of clusters of data, and the result is shown in Figure 2.

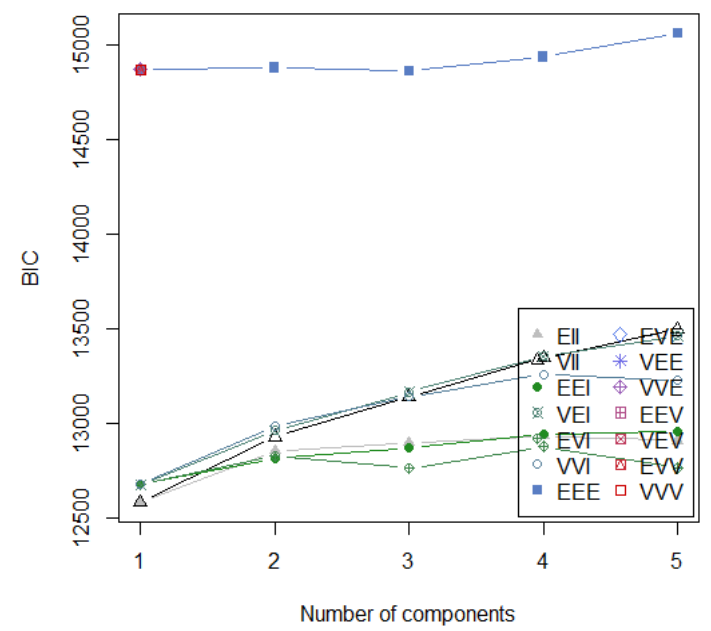

Fig.2. The result of cluster number determination of optimal fitting degree 
After the number of clusters is determined, the "hclust" package is used to cluster the mixed data. In this paper, the average distance between clusters is used as the principle to measure the mounting distance, which is expressed by "average". Combined with $\mathrm{R}$, the typical load curve is extracted from the complex chaotic data, and Results of DIANA clustering of load data of Liaoning province in 2017 is shown in Figure 3.

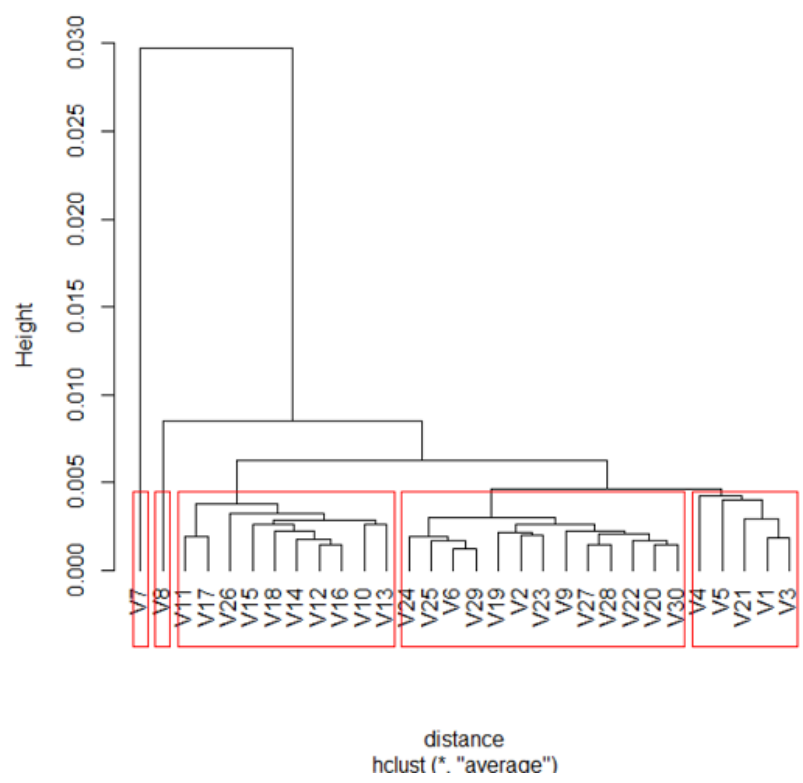

Fig.3. Results of DIANA clustering

Compared with the Diana method, DBSCAN is not sensitive to the order of samples in the database and can find clusters of any shape, which has the function of identifying noise points. First of all, the optimal scanning radius should be determined. By drawing the k-distance curve, the inflection point should be found, that is, the obvious inflection point position is the corresponding better parameter, and the appropriate scanning radius value should be found. This step is implemented by using the knndistplot function, and the optimal scan radius value selected is shown in Figure 4 based on the k-distance curve.

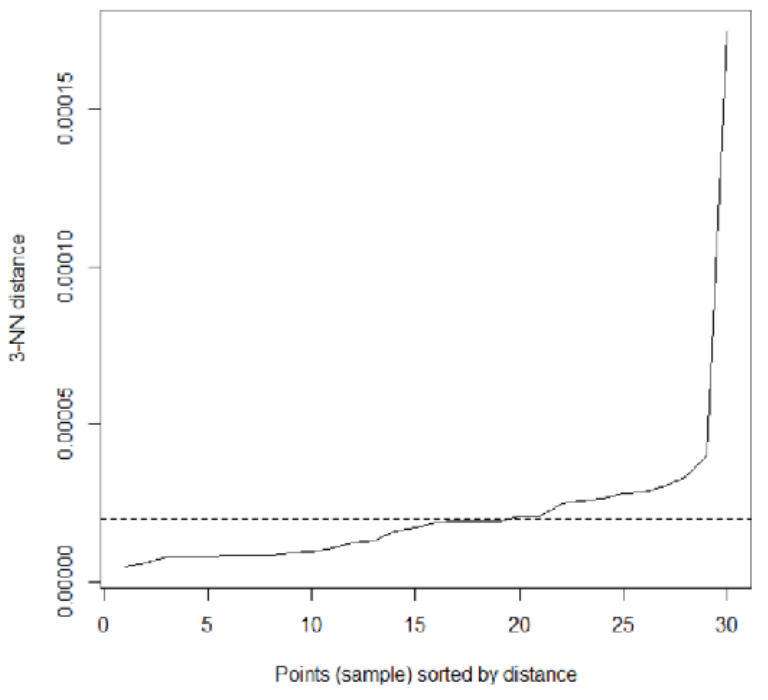

Fig.4. The optimal scan radius value selected

Knndistplot calculates the distance of the nearest neighbor in the point matrix, and then sorts the distance from small to large, and displays it in a graph. The $\mathrm{x}$-axis is the sequence number of the distance, and the $y$-axis is the value of the distance. Find out the obvious inflection point on the k-distance curve through human eye recognition, draw a dotted line parallel to the $\mathrm{x}$-axis with $y=0.00003$, and highlight the mark. Therefore, the final confirmed scanning radius EPS is 0.00003 . Next, the DBSCAN function is called to cluster the data set, and DBSCAN clustering results of load data of Liaoning province in 2017 are shown in Figure 5.

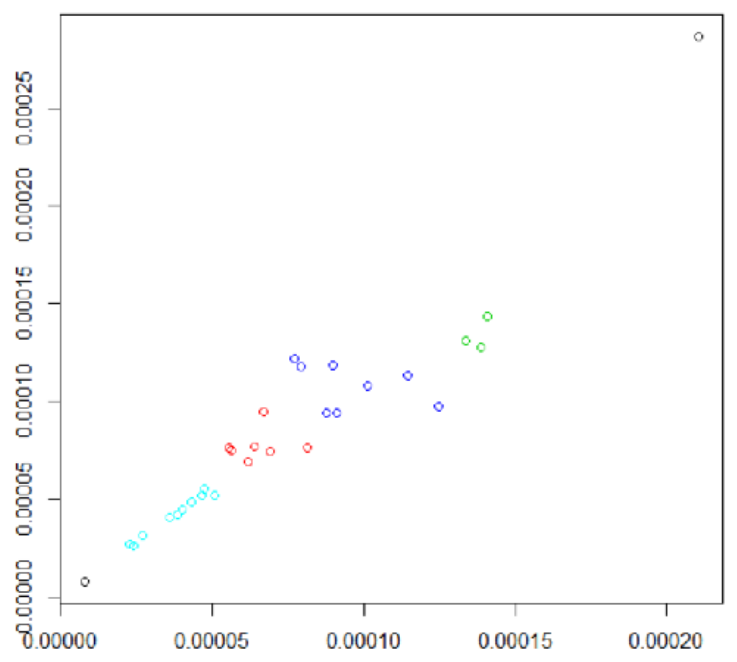

Fig.5. DBSCAN clustering results

\section{B. Power Consumption and Economic Relationship}

Taking the monthly electricity consumption of a University Park in North China in 2017 as an example, this paper analyzes the impact of months on electricity consumption from two aspects: the relationship between electricity consumption and holidays, and the relationship between electricity consumption and seasons. The monthly electricity consumption curve of a University Park in North China is shown in Figure 6.

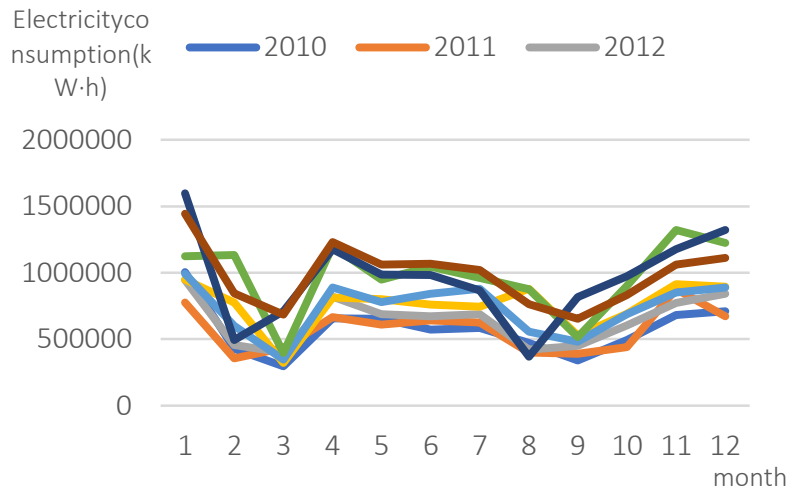

Fig.6. Monthly electricity consumption curve of a university park in Shenyang from 2010 to 2017

$Y_{d}$ is the time series object obtained by logarithmic transformation, $Y_{t}$ is the trend component, $Y_{s}$ is the seasonal component, $Y_{r}$ is the random component. Taking March and April as examples, the stl function in $\mathrm{R}$ language is used to draw the change curve of time series object $Y_{d}$, and it is decomposed into corresponding $Y_{t}, Y_{s}$ and $Y_{r}$. the change trend of each component is shown in Figure 7. 

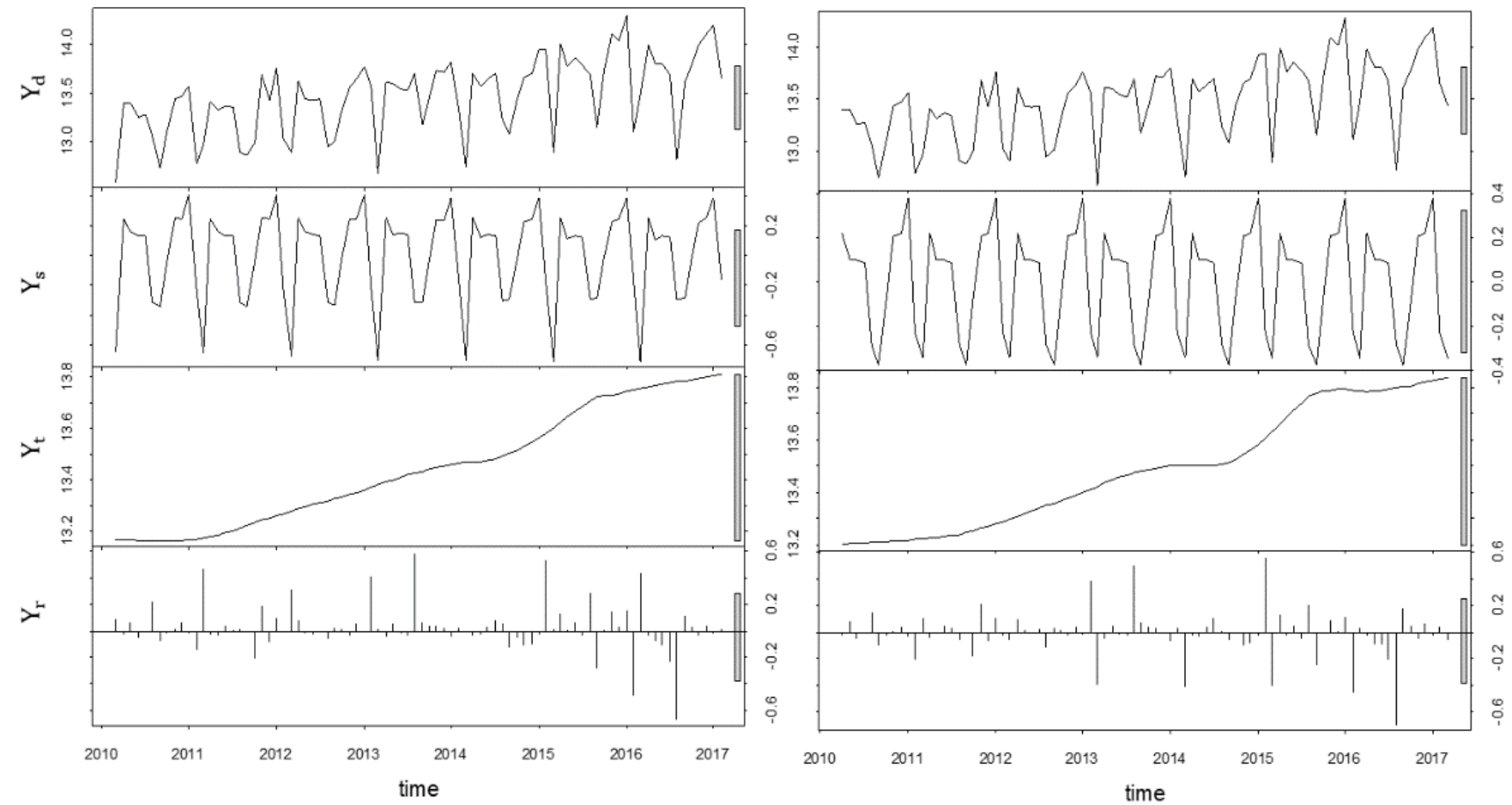

Fig.7. March (left) and April (right) trends of original sequence with its components

\section{Eviews Simulation}

It can be seen that the seasonal component $Y_{s}$ of the month with seasonal mutation changes gradually with time, but the seasonal component $Y_{s}$ of the month without inflection point changes periodically, $u$ has a stable growth trend, $Y_{r}$ has no development rule.

LNGDP is Liaoning GDP. In order to ensure that the sample size of economic factors is consistent with the monthly electricity consumption, the GDP of each quarter is divided into months, and the approximate monthly GDO data is obtained. Read in the monthly GDP data of a city in the north, decompose it with X12 model, and click Proc / Seasonal Adjustment / X12 to get the trend component data of GDP. In order to ensure the stability of the data, we can take the natural logarithm of the trend component, process the data, and then build the VAR model. The trend component of GDP is shown in Figure 8.

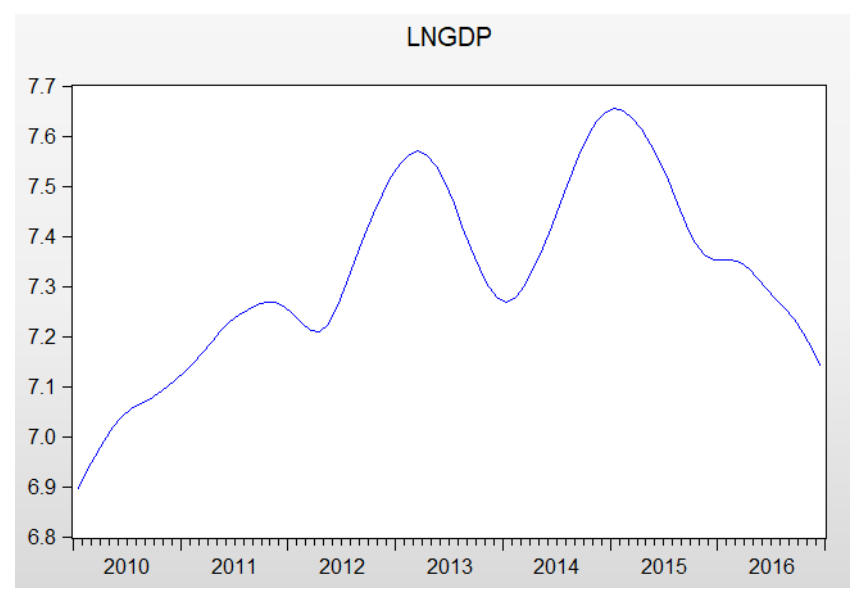

Fig.8. GDP trend component curve
Open the trend components of GDP and electricity consumption in var package, click Lag Length criteria, and the confidence interval of lag order will pop up as shown in Table I .

TABLE I. The confidence interval for the lag order of VAR model

\begin{tabular}{|c|c|c|c|c|c|c|}
\hline Lag & LogL & LR & FPE & AIC & SC & HQ \\
\hline 0 & -919.8341 & NA & $4.53 \mathrm{e}+08$ & 25.60650 & 25.66974 & 25.63168 \\
\hline 1 & -519.9274 & 766.4878 & 7581.285 & 14.60910 & 14.79882 & 14.68462 \\
\hline 2 & -392.8206 & 236.5599 & 248.1805 & 11.18946 & 11.50567 & 11.31534 \\
\hline 3 & -322.0301 & 127.8163 & 38.84761 & 9.334168 & 9.776853 & 9.510403 \\
\hline 4 & -304.8392 & 30.08404 & 26.96743 & 8.967755 & 9.536922 & 9.194342 \\
\hline 5 & -295.4543 & 15.90214 & 23.27183 & 8.818175 & 9.513823 & 9.095115 \\
\hline 6 & -293.7491 & 2.794622 & 24.88266 & 8.881920 & 9.704049 & 9.209212 \\
\hline 7 & -277.5902 & 25.58493 & 17.82851 & 8.544173 & $9.492784 *$ & $8.921818 *$ \\
\hline 8 & -273.6755 & 5.980746 & 17.97444 & 8.546543 & 9.621635 & 8.974540 \\
\hline 9 & -273.2689 & 0.598628 & 20.00905 & 8.646359 & 9.847933 & 9.124709 \\
\hline 10 & -266.7881 & 9.181128 & 18.85117 & 8.577448 & 9.905503 & 9.106151 \\
\hline 11 & -257.7192 & $12.34380 *$ & $16.56391 *$ & $8.436645 *$ & 9.891182 & 9.015701 \\
\hline 12 & -255.9084 & 2.364098 & 17.84887 & 8.497456 & 10.07847 & 9.126864 \\
\hline
\end{tabular}

LR: sequential modified LR test statistic. FPE: Final prediction error. AIC: Akaike information criterion. SC: Schwarz information criterion. HQ: Hannan-Quinn information criterion. The order of hysteresis is represented by $\mathrm{p}(1 \ll \mathrm{P} \ll \mathrm{n}, \mathrm{n}$ is the sample size $)$. Because the delay of monetary policy is generally $6-12$ months, this section sets the upper limit of the lag order as 12 .

As can be seen from Table I, when $\mathrm{p}=11$, the evaluation 
index is the best, so the lag order is determined as 11 .

VAR model is effective to predict the interconnected time series variable system. If there is no correlation between variables, it is not suitable to build VAR model. In order to determine whether GDP changes play a major role in electricity consumption, this section conducts exogenous tests on two variables. Click View / lag structure / Granger causality to pop up the exogeneity test results as shown in Table II and Table III.

TABLE II. Dependent variable: LN GDP

\begin{tabular}{|c|c|c|c|}
\hline Excluded & Chi-sq & df & Prob \\
\hline GDP_HP & 30.56972 & 11 & 0.0013 \\
\hline All & 30.56972 & 11 & 0.0013 \\
\hline
\end{tabular}

TABLE III. Dependent variable: GDP_HP

\begin{tabular}{|c|c|c|c|}
\hline Excluded & Chi-sq & df & Prob \\
\hline GDP_HP & 23.12329 & 11 & 0.0170 \\
\hline All & 23.12329 & 11 & 0.0170 \\
\hline
\end{tabular}

From the table, it can be seen that the Prob value is less than 0.05 , that is, there is no exogeneity, indicating that GDP has a significant impact on electricity consumption, that is, it has the ability to predict, and it is meaningful to establish VAR model.

First, the model parameters are estimated. Open the VAR setting box of GDP and electricity consumption data, click $\mathrm{OK}$, and the estimated result window will pop up as shown in Table IV.

TABLE IV. VAR parameter estimation results

\begin{tabular}{|c|c|c|}
\hline & LNGDP & POWER_T \\
\hline \multirow{3}{*}{ GDP_HP(-1) } & 0.892644 & -0.318902 \\
& $(0.11730)$ & $(0.30656)$ \\
& {$[7.61004]$} & {$[-1.04026]$} \\
\hline \multirow{2}{*}{ GDP_HP(-2) } & -0.025358 & 0.608604 \\
& $(0.12315)$ & $0.32184)$ \\
& {$[-0.20592]$} & {$[1.89100]$} \\
\hline \multirow{2}{*}{ POWER_HP(-1) } & -0.020718 & -0.160956 \\
& $(0.04430)$ & $0.11577)$ \\
\hline \multirow{3}{*}{ POWER_HP(-2) } & {$[-0.46773]$} & {$[-1.39035]$} \\
& 0.005056 & 0.036296 \\
& $(0.04195)$ & $(0.10965)$ \\
\hline \multirow{2}{*}{ c } & {$[0.12052]$} & {$[0.33102]$} \\
& -0.001664 & -0.006855 \\
& $(0.00618)$ & $(0.01615)$ \\
& {$[0.26925]$} & {$[0.42438]$} \\
\hline
\end{tabular}

\section{CONCLUSION}

Based on the autocorrelation of monthly electricity consumption time series, the influence of season mutation and major holidays on electricity consumption prediction is considered from the perspective of time. A comprehensive forecasting method of monthly electricity consumption based on STL decomposition model is proposed, which makes full use of the decomposition characteristics of STL decomposition model. According to the change rate of seasonal components of electricity consumption in different months, periodic decomposition and non-periodic decomposition are adopted respectively, and then appropriate models are selected to predict each component respectively, which provides an effective forecasting idea for monthly electricity consumption prediction. On the basis of considering the influence of seasonal sudden change and major holidays on monthly electricity consumption, this paper proposes improvement measures for the prediction method of monthly electricity consumption based on the correlation between electricity consumption and economic development. Firstly, STL model and X12 model are used to decompose the power consumption sequence and GDP sequence respectively, and the trend component of GDP is taken as the influencing factor of monthly power consumption trend component into VAR model to forecast the power consumption trend; then BP neural network is used to forecast the seasonal component and average method is used to forecast the random component. Using the actual data of a University Park in the north of China to simulate and analyze the monthly electricity consumption forecasting method considering the influence of economic factors, it is proved that the method is accurate and effective.

\section{REFERENCES}

[1]. Ghalehkhondabi I, Ardjmand E, Weckman GR, and William A, “An overview of energy demand forecasting methods published in 20052015," Energy Systems, vol. 8, no. 12, pp. 411-447, 2017.

[2]. Hamzaçebi C, Es HA, and Çakmak R, "Forecasting of Turkey's monthly electricity demand by seasonal artificial neural network," Neural Computing \& Applications, vo. 33, no. 3, pp. 71-76, 2017.

[3]. C Vu DH, Muttaqi KM, and Agalgaonkar AP, "A variance inflation factor and backward elimination based robust regression model for forecasting monthly electricity demand using climatic variables," Applied Energy, no. 140, pp. 385-394, 2015.

[4]. De Felice M, Alessandri A, and Ruti PM, "Electricity demand forecasting over Italy potential benefits using numerical weather prediction models," Electric Power System, vol. 104, pp. 71-79, 2013.

[5]. Pachauri RK, Allen MR, Barros VR, et al, "Synthesis report. contribution of working groups I, II and III to the fifth assessment report of the intergovernmental panel on climate change," Journal of Romance Studies, vol. 4, no. 2, pp. 85-88, 2014.

[6]. Y.X. He, J. Jiao, Q. Chen, S.F. Ge,Y. Chang, and Y. Xu, "Urban long term electricity demand forecast method based on system dynamics of the new economic normal: The case of Tianjin," Energy, vol. 133, no. 31, pp. 9-22, 2017.

[7]. Z.B. Wu, J.P. Xu, "Predicting and optimization of energy consumption using system dynamics-fuzzy multiple objective programming in world heritage areas," Energy, vol. 49, no. 4, pp. 19-31, 2013.

[8]. Y. Yuan, K. Dehghanpour, F. Bu and Z. Wang, "A multi-timescale data-driven approach to enhance distribution system observability," IEEE Transactions on Power Systems, vol. 34, no. 4, pp. 3168-3177, July 2019.

[9]. Paudel S., Elmtiri M., Kling W., LeCorre O., Lacarrière B., "Pseudo dynamic transitional modeling of building heating energy demand using artificial neural network," Energy \& Buildings, vol. 70, no. 2, pp. 81-93, 2014.

[10]. Adamowski J, Chan H F, Prasher S O, Ozga - Zielinski B, and Sliusarieva A, "Comparison of multiple linear and nonlinear regression, autoregressive integrated moving average, artificial neural network, and wavelet artificial neural network methods for urban water demand forecasting in Montreal, Canada," Water Resources Research, vol. 48, no. 1, pp. 273-279, 2012. 